\title{
The Involvement of Phosphatase and Tensin Homolog Deleted on Chromosome Ten (PTEN) in the Regulation of Inflammation Following Coronary Microembolization
}

\author{
Jiangyou Wang ${ }^{\mathrm{a}}$ Lang Lia Qiang Su ${ }^{\mathrm{a}}$ You Zhou ${ }^{\mathrm{a}}$ Han Chen ${ }^{\mathrm{b}}$ Guotian Ma \\ Tao Liu ${ }^{a}$ Zhongli Tang ${ }^{a}$ Yangchun Liu ${ }^{a}$ \\ aDepartment of Cardiology, the First Affiliated Hospital of Guangxi Medical University, Nanning, \\ bDepartment of Pediatric, Wuhan Asia Heart Hospital, Wuhan, China
}

\section{Key Words}

Coronary microembolization $\bullet$ Inflammation $\bullet$ TNF- $\alpha \cdot$ PTEN

\begin{abstract}
Background/Aims: Growing evidence shows that phosphatase and tensin homolog deleted on chromosome ten (PTEN) is involved in regulating inflammation in different pathological conditions. Therefore, we hypothesized that the upregulation of PTEN correlates with the impairment of cardiac function in swine following coronary microembolization (CME). Methods: To possibly disclose an anti-inflammatory effect of PTEN, we induced swine CME by injecting inertia plastic microspheres ( $42 \mu \mathrm{m}$ in diameter) into the left anterior descending coronary artery and analyzed the myocardial tissue by immunochemistry, qRT-PCR and western blot analyses. In addition, we downregulated PTEN using siRNA. Results: Following CME, PTEN mRNA and protein levels were elevated as early as $3 h$, peaked at $12 h$, and then continuously decreased at $24 \mathrm{~h}$ and $48 \mathrm{~h}$ but remained elevated. Through linear correlation analysis, the PTEN protein level positively correlated with cTnI and TNF- $\alpha$ but was negatively correlated with LVEF. Furthermore, PTEN siRNA reduced the microinfarct volume, improved cardiac function (LVEF), reduced the release of CTnI, and suppressed PTEN and TNF- $\alpha$ protein expression. Conclusion: This study demonstrated, for the first time, that PTEN is involved in CME-induced inflammatory injury. The data generated from this study provide a rationale for the development of PTEN-based anti-inflammatory strategies.
\end{abstract}




\section{Introduction}

Coronary microembolization (CME) is a major pathogenic outcome of acute myocardial infarction (MI) and is characterized by the spontaneous embolization of thrombotic material and debris or iatrogenic rupture of atherosclerotic plaque-induced obstructions of the coronary microvascular system [1-3]. Accordingly, the resulting coronary microcirculation dysfunction might be determined by perfusion-contraction mismatch, reactive hyperemia, and regional myocardial contractile dysfunction; however, myocardial function partially recovers within minutes $[4,5]$. Many studies have shown that CME-induced microinfarcts initiate an inflammatory reaction with increased tumor necrosis factor- $\alpha$ (TNF- $\alpha$ ) expression [6,7], resulting in damage to the myocardium and deterioration of myocardial systolic function $[8,9]$. Moreover, our previous studies also verified that microinfarction and ischemia in microembolized myocardium cause a severe inflammatory response with increased TNF- $\alpha$ expression in concert with the impairment of cardiac function $[10,11]$.

Phosphatase and tensin homolog deleted on chromosome ten (PTEN) dephosphorylates PI-3,4,5-trisphosphate (PIP3) to PI-4,5-bisphosphate (PIP2) and Pi to antagonize the actions of phosphatidylinositol (PI)-3-kinase (PI3K) [12]. Inhibition of PTEN by drugs or knockdown of cardiac-specific PTEN reinforces PI3K activity, inhibits apoptosis, reduces the infarct area, and ameliorates cardiac injury after ischemia-reperfusion [13]. Furthermore, inactivation of cardiac-specific PTEN protects against cardiac failure and myocardial fibrosis in a hypertensive mouse model [14]. PTEN expedites the inflammation response in post-MI remodeling but mitigates the inflammation response in vascular endothelial repair $[9,15]$. Nevertheless, the role of PTEN in post-CME myocardial injury has not yet been studied.

Therefore, we focused on the dynamic changes in PTEN expression in myocardium post-CME and concluded that the causal relationship between PTEN and TNF- $\alpha$ exists to prevent post-CME myocardial injury.

\section{Materials and Methods}

\section{Animal preparation}

Forty miniature swine of either sex (body weight $25-30 \mathrm{~kg}$ ) were supplied by the Animal Center of the College of Agriculture, Guangxi University (Nanning, People's Republic of China). Throughout all experimental stages, the animals were kept under controlled conditions for temperature, humidity, and light, with pig feed and water available ad libitum. The investigation conformed to the Guide for the Care and Use of Laboratory Animals published by the US National Institutes of Health (NIH publication No. 85-23, revised 1996). The Clinical and Animal Research Ethics Committees of Guangxi Medical University approved all procedures, and the number and suffering of the animals were minimized according to the guidelines of the American Physiological Society.

\section{Swine models for CME and treatment groups}

Fifty miniature swine of either sex (body weight 25-30 kg) were randomly assigned to ten groups as follows: the Sham surgery groups $(n=20)$, which were euthanized at $3 h(n=5), 12 h(n=5), 24 h(n=5)$, and $48 \mathrm{~h}(\mathrm{n}=5)$; the CME groups $(\mathrm{n}=20)$, which were euthanized at $3 \mathrm{~h}(\mathrm{n}=5), 12 \mathrm{~h}(\mathrm{n}=5), 24 \mathrm{~h}(\mathrm{n}=5)$, and 48 $\mathrm{h}(\mathrm{n}=5)$; the CME pre-treated with PTEN siRNA group, which was euthanized at $12 \mathrm{~h}(\mathrm{n}=5)$; and the CME pre-treated with control siRNA group, which was euthanized at $12 \mathrm{~h}(\mathrm{n}=5)$. The CME model was induced by manual unremitting injection of microspheres into the left anterior descending (LAD) artery, as previously described by Carlsson et al. [16]. Briefly, the animals were initially sedated with intramuscular injection of a combination of ketamine $(10-15 \mathrm{mg} / \mathrm{kg}$ ) and atropine $(2 \mathrm{mg})$. After endotracheal intubation, anesthesia was maintained by intravenous administration of diazepam $(5 \mathrm{mg} / \mathrm{h})$ into the ear vein. The right femoral artery was dissected, and a $6 \mathrm{~F}$ vascular sheath (Johnson \& Johnson-Cordis Corporation, Miami, FL, USA) was placed in the artery. Prior to coronary cannulation, the animals were anticoagulated with intravenous sodium heparin (200 U/kg), and an additional dose of $100 \mathrm{U} / \mathrm{kg}$ was given at hourly intervals to maintain heparinization. A 6F JL 4.0 guiding catheter was used for the left coronary artery system via the right femoral 
approach for coronary angiography, and a $1.8 \mathrm{~F}$ infusion catheter (Johnson \& Johnson-Cordis Corporation, USA) was then placed in the LAD artery with the tip between the second and third diagonal branches. Microembolization was induced by manual unremitting injection of 100,000 microspheres with a diameter of $42 \mu \mathrm{m}$ (Dynospheres; Dyno Particles, Lillestrøm, Norway) into the LAD artery within 40 minutes followed by a $10-\mathrm{mL}$ saline flush. For the sham surgery, the identical procedure was performed, but normal saline was substituted for the microsphere suspension. The systemic blood pressure and heart rate were continuously monitored during the procedure. Twenty swine were subjected to sham surgery $(n=20)$, and twenty swine underwent the previously described procedures to create a CME model $(n=20)$. The swine from the CME or Sham surgery groups were euthanized at $3 \mathrm{~h}, 12 \mathrm{~h}, 24 \mathrm{~h}$, and $48 \mathrm{~h}$ post-operatively, resulting in 4 subgroups each ( $n=5$ each) and yielding 8 subgroups $(n=5$ each). Because all biomarkers in our preliminary experiment had the maximal alteration when animals were sacrificed at $12 \mathrm{~h}$, the swine in the CME previously treated with PTEN siRNA $(n=5)$ and CME previously treated with control siRNA $(n=5)$ groups were minimized to five and sacrificed at $12 \mathrm{~h}$. In vivo transfer was performed according to the method described previously [17]. Briefly, the animals in these two groups were handled exactly like the CME model swine, except that $100 \mu \mathrm{g}$ of PTEN siRNA (PTEN-sus-368; GenePharma, shanghai, China) or control siRNA (GenePharma) was diluted in the same volume of in vivo transfection reagent (Entranster ${ }^{\mathrm{TM}}$-in vivo; Engreen, Beijing, China), mixed gently by pipetting the solution up and down, and selectively infused into the LAD artery within 1 minute with a flush of $2 \mathrm{~mL}$ of saline after the $1.8 \mathrm{~F}$ infusion catheter (Cordis Inc., USA) was placed in the LAD artery. The CME group pre-treated with PTEN siRNA was then created $72 \mathrm{~h}$ after in vivo PTEN siRNA transfer.

\section{Echocardiography}

The animals were sedated as previously described and placed on the experimental platform in the right lateral position. One experienced investigator who was blinded to the study protocol performed the transthoracic echocardiography using a GE VIVID 7 system with a 1.5-4.3 MHZ transducer. Briefly, the 1.5-4.3 MHz transducer was placed on the left anterior chest wall to obtain the left ventricle end-diastolic dimension (LVEDD), fractional shortening (FS), and cardiac output (CO), and the left ventricle ejection fraction (LVEF) was calculated using a cubic formula. All parameters were averaged from more than three consecutive cardiac cycles. After functional measurement, the animals were sacrificed by intravenous injection of $10 \mathrm{~mL}$ of $10 \%$ potassium chloride, and the hearts were fixed in $4 \%$ paraformaldehyde or quickly frozen at $-80^{\circ} \mathrm{C}$ for use.

\section{Coronary sinus levels of cTnI}

EDTA-anticoagulated blood samples were collected from the coronary sinus before the animals were sacrificed. After collection, blood samples were immediately subjected to centrifugation at $4000 \mathrm{rpm}$ for 15 min, and serum samples were stored at $-80^{\circ} \mathrm{C}$ until postmortem analysis. Measurement of the serum cTnI concentration was performed using commercially available electrochemical luminescence kits according to the manufacturer's instructions (Roche, Inc., Switzerland). All measurements were determined in duplicate.

\section{Tissue sampling}

After blood sample collection, the hearts were arrested in diastole by the injection of $10 \mathrm{~mL}$ of $10 \%$ potassium chloride into the ear vein. The heart was immediately removed along with the atrial appendage and the atrium cordis, and the left ventricle was separated into apex and base segments from the midpoint of the left ventricle long axis in a plane parallel to the atrioventricular groove. The apex was rapidly frozen in liquid nitrogen and stored at $-80^{\circ} \mathrm{C}$ until preparation for real-time PCR and western blot analysis. The base was fixed in $4 \%$ paraformaldehyde for $12 \mathrm{~h}$, embedded with paraffin, and serially sectioned ( $4 \mu \mathrm{m}$ thick; $\geq 12$ sections per animal). Three sections containing the same number of microinfarct foci were selected and then stained using Mayer's hematoxylin and eosin (H\&E). Hematoxylin-basic fuchsin picric acid (HBFP) staining was used to assess the myocardial microinfarction area on triplicate sections.

\section{Measuring the myocardial microinfarction area}

HBFP staining is an important method for the early diagnosis of myocardial ischemia. This stain dyes ischemic cardiac muscle, normal myocardial cytoplasm, and nuclei red, yellow, and blue, respectively. A DMR-Q550 pathological image pattern analysis instrument (Leica, Germany) was used to analyze the 
HBFP-stained sections. Briefly, five microscopic visual fields (original magnification $\times 100$ ) were randomly sampled from each section for observation using the QWin analysis software (Leica, Germany), and the planar area method was used to measure the infarction zone, which was expressed as a percentage of the area of the bulk analysis section and averaged. The relative ischemic area was calculated according to the following formula: ischemic area/total area $\times 100 \%$.

\section{Real-time quantitative PCR analysis}

Total RNA samples from cardiac tissue blocks were isolated using the TRIzol Reagent kit (GIBCO, USA) according to the manufacturer's protocols. Total RNA (4 $\mu$ g) was then reverse transcribed using a Reverse Transcriptase Kit (Promega, USA) according to the manufacturer's instructions. Real-time PCR was performed on the ABI PRISM 7000 system (Applied BioSystems, CA, USA) using the sequence-nonspecific SYBR Green I dye (TaKaRa, Japan) for 40 cycles. PTEN and TNF- $\alpha$ in cardiomyocytes were detected using qRTPCR analysis, with actin as an internal control. PCR was performed in a $25-\mu$ l reaction mixture containing $2 \mu \mathrm{l}$ of cDNA, primers ( $0.5 \mu \mathrm{l}$ each), and $12.5 \mu \mathrm{l}$ of the SYBR Green Master Mix (Applied BioSystems, CA, USA), with water added to the final volume. PCR was initiated by an initial denaturation step at $95^{\circ} \mathrm{C}$ for 10 minutes; 40 cycles of denaturation at $95^{\circ} \mathrm{C}$ for 15 seconds, annealing at $60^{\circ} \mathrm{C}$ for 30 seconds, and extension at $72^{\circ} \mathrm{C}$ for 15 seconds; a final extension at $95^{\circ} \mathrm{C}$ for 15 seconds; and then 1 cycle of dissociation at $60^{\circ} \mathrm{C}$ for 1 minute; and a final dissociation at $95^{\circ} \mathrm{C}$ for 15 seconds. The primers were 5 -GAG CCA TTT CCA TCC TGC AG-3 and 5-GCT GTC ATG TCT GGG AGT CT-3 for swine PTEN, 5-GGC CCA AGG ACT CAG ATC AT-3 and 5-GCA TAC CCA CTC TGC CAT TG-3 for swine TNF- $\alpha$, and 5-CAC CTT CTA CAA CGA GCT GC-3 and 5-TCA TCT TCT CAC GGT TGG CT-3 for actin (all primers were designed by TaKaRa, Japan). The transcript expression levels were quantified using the Ct value method, where values were normalized to actin as an internal control in the same sample. The specificity of the PCR product amplification was confirmed by their respective melting curves.

\section{Western blot analysis}

Tissue blocks (10 mg) of the ventricle anterior wall were homogenized in RIPA lysis buffer ( $50 \mathrm{mmol} / \mathrm{L}$ Tris $\mathrm{HCl} \mathrm{pH} \mathrm{7.4,} \mathrm{1 \%} \mathrm{NP-40,} \mathrm{0.25 \%} \mathrm{sodium} \mathrm{deoxycholate,} 150 \mathrm{mmol} / \mathrm{L} \mathrm{NaCl}, 1 \mathrm{mmol} / \mathrm{L}$ EDTA, and protease and phosphatase inhibitors) on ice for 30 seconds. Tissue suspensions were incubated on ice for 10 minutes and centrifuged at 10,000 rpm for 10 minutes. Briefly, the protein concentrations were determined with a bicinchoninic acid protein assay kit using bovine serum albumin as the standard. Equal amounts of protein $(100 \mu \mathrm{g})$ were fractionated by sodium dodecyl sulfate-polyacrylamide gel electrophoresis (SDS-PAGE) and blotted onto polyvinylidene (PVDF) membranes (Millipore, Bedford, MA, USA). The membranes were blocked for $2 \mathrm{~h}$ using 5\% non-fat milk in Tris-buffered saline supplemented with Tween-20 (TBST) and probed overnight at $4^{\circ} \mathrm{C}$ with the following primary antibodies: PTEN (1:1000 dilution, Aviva Systems Biology), TNF- $\alpha$ (1:1000 dilution, Abcam), and anti-glyceraldehyde-3-phosphate dehydrogenase (GAPDH) (1:1000 dilution, PTG). The membranes were then rinsed with TBST and incubated with horseradish peroxidaseconjugated secondary anti-GAPDH (1:8000 dilution, Alexa Fluor ${ }^{\circledR} 700$ goat anti-rabbit IgG [H+L], or Alexa Fluor® 800 goat anti-rabbit IgG [H+L], Invitrogen) at room temperature for $1 \mathrm{~h}$. Horseradish peroxidaselabeled anti-GAPDH (1:1000) served as an internal control. The blots were exposed to X-ray film, and the integrated absorbance of the identified bands was quantified using the Bio-Rad imaging software (Bio-Rad, Hercules, CA, USA).

\section{Statistical analysis}

All quantitative data are expressed as the means (SD) and were analyzed using the SPSS 13.0 software. Two-tailed, unpaired Student's t-tests, one-way ANOVA, and Pearson correlation analysis were used for statistical evaluation of the data. Differences were considered statistically significant when $p<0.05$.

\section{Results}

Animal groups

No statistically significant differences $(p>0.05)$ in body weight or heart rate were identified between all groups (data not shown). 
Table 1. Changes in cTnI before and after CME (ng/mL). Data are presented as the means (SD); a, $p<0.05$ compared with the Sham group; b, $p<0.05$ compared with the CME group at $12 \mathrm{~h}$;,$p<0.05$ compared with pre-operation $(0 \mathrm{~h})$

\begin{tabular}{lcclll}
\hline Parameters & \multicolumn{1}{c}{$0 \mathrm{~h}$} & \multicolumn{1}{c}{$3 \mathrm{~h}$} & \multicolumn{1}{c}{$12 \mathrm{~h}$} & \multicolumn{1}{c}{$24 \mathrm{~h}$} & \multicolumn{1}{c}{$48 \mathrm{~h}$} \\
\hline Sham (cTnI) & $0.041(0.003)$ & $0.043(0.002)$ & $0.042(0.003)$ & $0.042(0.003)$ & $0.043(0.004)$ \\
CME (cTnI) & $0.042(0.002)$ & $0.100(0.003)^{\mathrm{abc}}$ & $0.226(0.013)^{\mathrm{ac}}$ & $0.109(0.002)^{\mathrm{abc}}$ & $0.070(0.004)^{\mathrm{abc}}$ \\
\hline
\end{tabular}

Table 2. Changes in cardiac function after CME. Each point represents the mean value (SD). The variables were measured in swine by echocardiography at $3 \mathrm{~h}, 12 \mathrm{~h}, 24 \mathrm{~h}$, and 48 $\mathrm{h}$ after CME or Sham surgery. a, $\mathrm{p}<0.05$ compared with the Sham group; and b, p<0.05 compared with the CME group at $12 \mathrm{~h}$. LVEF, left ventricular ejection fraction; FS, fractional shortening; LVEDD, left ventricular end-diastolic diameters; CO, cardiac output

Fig. 1. cTnI concentrations before $(0 \mathrm{~h})$ and after CME in the CME and Sham groups at 3 h, 12 h, 24 h, and 48 h. The arrow indicates the injection of embolizing microspheres or placebo into the CME or Sham groups, respectively. a, $p<0.05$ compared with the Sham group; $\mathrm{b}, p<0.05$ compared with the CME group at $12 \mathrm{~h}$.

\begin{tabular}{|c|c|c|c|c|}
\hline Variables & S LVEF $(\%)$ & FS (\%) & LVEDD (mm) & $\overline{\mathrm{CO}(\mathrm{L} / \mathrm{min})}$ \\
\hline \multicolumn{5}{|c|}{ Sham } \\
\hline $3 \mathrm{~h}$ & $67.10(1.25)$ & $43.22(1.87)$ & $31.56(1.68)$ & $4.56(0.98)$ \\
\hline $12 \mathrm{~h}$ & $66.76(2.45)$ & $41.35(1.58)$ & $32.82(1.83)$ & $4.29(0.79)$ \\
\hline $24 \mathrm{~h}$ & 6282 & $42.84(1.89)$ & 31 & 4.4 \\
\hline $\begin{array}{l}48 \mathrm{~h} \\
\mathrm{CME}\end{array}$ & $67.48(1.72)$ & $40.38(1.93)$ & 32.84 & 4.2 \\
\hline $3 \mathrm{~h}$ & \multicolumn{4}{|c|}{ 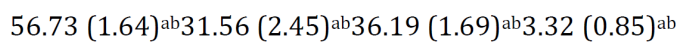 } \\
\hline $12 \mathrm{~h}$ & \multicolumn{4}{|c|}{$48.14(1.95)^{\mathrm{a}} 22.94(1.54)^{\mathrm{a}} 39.38(2.23)^{\mathrm{a}} 2.54(0.63)^{\mathrm{a}}$} \\
\hline $24 \mathrm{~h}$ & \multicolumn{4}{|c|}{ 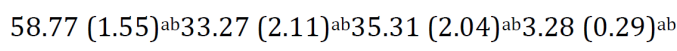 } \\
\hline $48 \mathrm{~h}$ & \multicolumn{4}{|c|}{$61.80(1.18)^{\mathrm{ab}} 37.63(2.36)^{\mathrm{ab}} 34.21(1.64)^{\mathrm{ab}} 3.35(0.76)^{\mathrm{ab}}$} \\
\hline
\end{tabular}

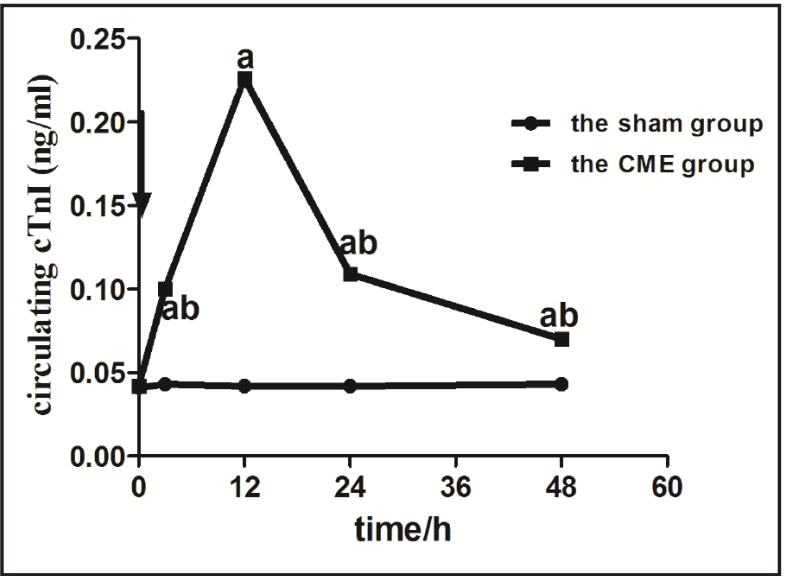

Serial change of cTnI before and after CME

As shown in Table 1 and Fig. 1, cTnI was significantly higher in the CME group than in the Sham group at each time point post-operation. cTnI rapidly increased as early as $3 \mathrm{~h}$ following the surgery, increased to the highest level at $12 \mathrm{~h}$, and then decreased continuously at $24 \mathrm{~h}$ and $48 \mathrm{~h}$ compared with the Sham group $(p<0.001)$.

Serial change of cardiac function after CME

Cardiac ultrasonography (Table 2) revealed that all parameters, except LVEDD, remained at a high level in the Sham group but were sharply reduced at $3 \mathrm{~h}$ and $12 \mathrm{~h}(p<0.05)$ and continuously increased at $24 \mathrm{~h}$ and $48 \mathrm{~h}$ in the CME group $(p<0.05)$. However, compared with the Sham group, significant increases in LVEDD were observed from 3 to $12 \mathrm{~h}$ after CME $(p<0.05)$, and LVEDD decreased at 24 and $48 \mathrm{~h}(p<0.05)$.

\section{CME histopathology}

Slight subendocardial ischemia without infarcts was observed in the Sham group (Fig. 2a). Multiple microinfarction regions were wedge-shaped, locally distributed, and nontransmural in the CME group (Fig. 2b), and these regions were mostly located in the subendocardium of the left ventricular anterior wall and apex of the heart. HE staining 


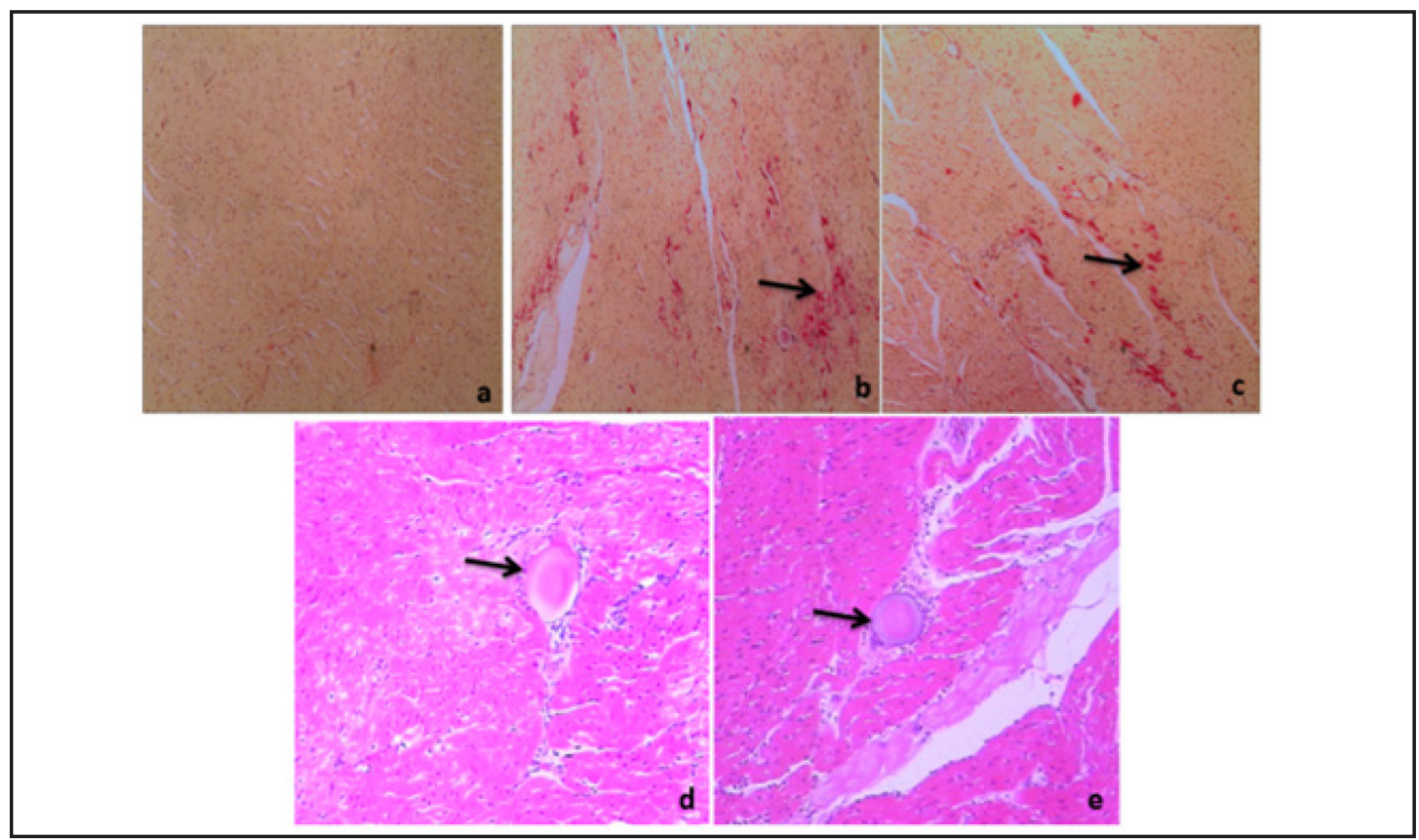

Fig. 2. (a, b, c) HBFP staining showed no necrotic myocardium in the Sham group (a), patchy necrotic myocardium at $12 \mathrm{~h}$ after CME (b), and funiform necrotic myocardium when the CME-treated swine were pretreated with PTEN siRNA (c). Necrosis is indicated by arrows. Normal myocardial cytoplasm, nuclei, and necrotic areas are stained yellow, blue, and red, respectively (HBFP staining, $\times 100)$. Figure 2 (d, e) H\&E staining showed myocardial necrotic foci with a high level of inflammatory cell infiltration $12 \mathrm{~h}$ after CME (d) and localized necrotic myocardium pre-transfected with PTEN siRNA after CME with low-level inflammatory cell infiltration (e). The arrow shows a 42- $\mu \mathrm{m}$ microsphere following the experimental protocol (H\&E staining; $\times 200$ ).

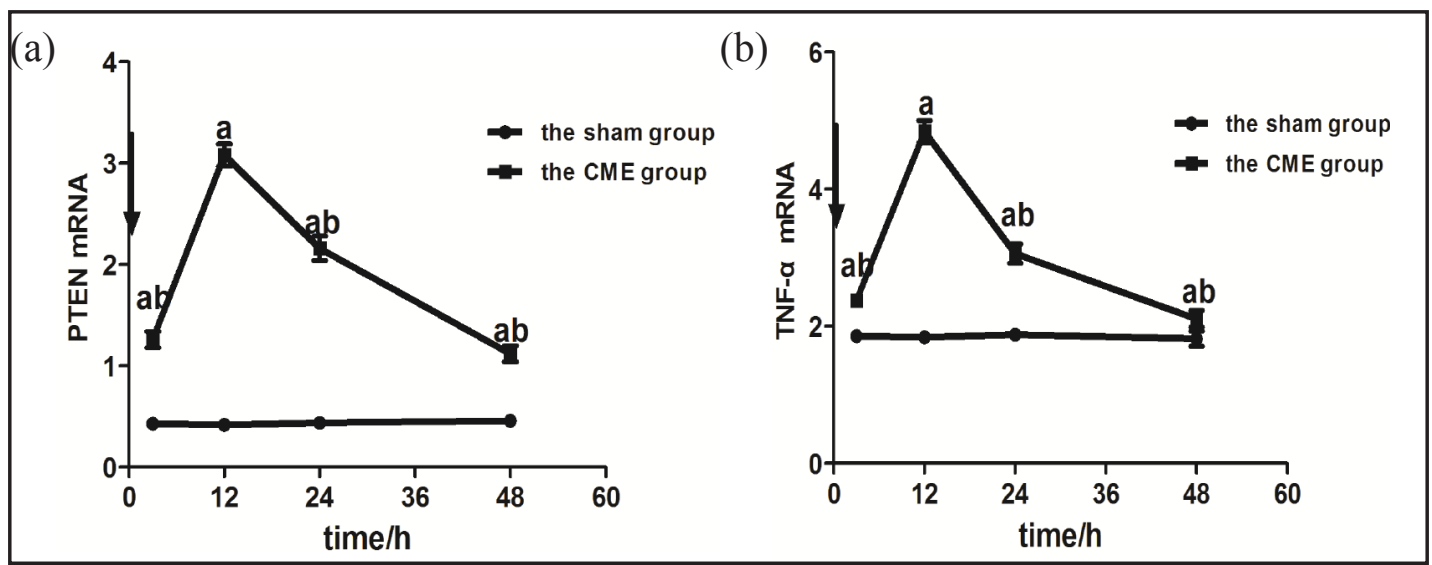

Fig. 3. (a, b) PTEN and TNF- $\alpha$ mRNA levels were measured in the CME and Sham groups at $3 \mathrm{~h}, 12 \mathrm{~h}$, $24 \mathrm{~h}$, and $48 \mathrm{~h}$. The arrows indicate the injection of embolizing microspheres or placebo in (a) and (b), respectively. a, $p<0.05$ compared with the Sham group; b, $p<0.05$ compared with the CME group at $12 \mathrm{~h}$.

revealed myocardial karyolysis or hypochromatosis with cytoplasmic red dyeing in the area of the microinfarction (Fig. 2d). Furthermore, peripheral cardiac muscle edema and denaturation, peripheral inflammatory cell infiltration, and erythrocyte effusion were also observed (Fig. 2d). The infarct areas after CME were 4.57 (1.38)\%, 4.84 (1.27)\%, 4.27 (1.31)\% and $4.36(1.58) \%$ at $3 \mathrm{~h}, 12 \mathrm{~h}, 24 \mathrm{~h}$, and $48 \mathrm{~h}$, respectively, with no significant differences among the groups. 
Wang et al.: PTEN is Involved in CME-Induced Myocardial Inflammatory Injury

Fig. 4. $(a, b)$ PTEN and TNF- $\alpha$ protein levels were determined using western blot analysis (normalized to GAPDH). Time tein expression in the CME and Sham groups at $3 \mathrm{~h}, 12 \mathrm{~h}, 24 \mathrm{~h}$, and 48 h. $^{*} p<0.001$ vs. Sham. course of PTEN and TNF- $\alpha$ pro-

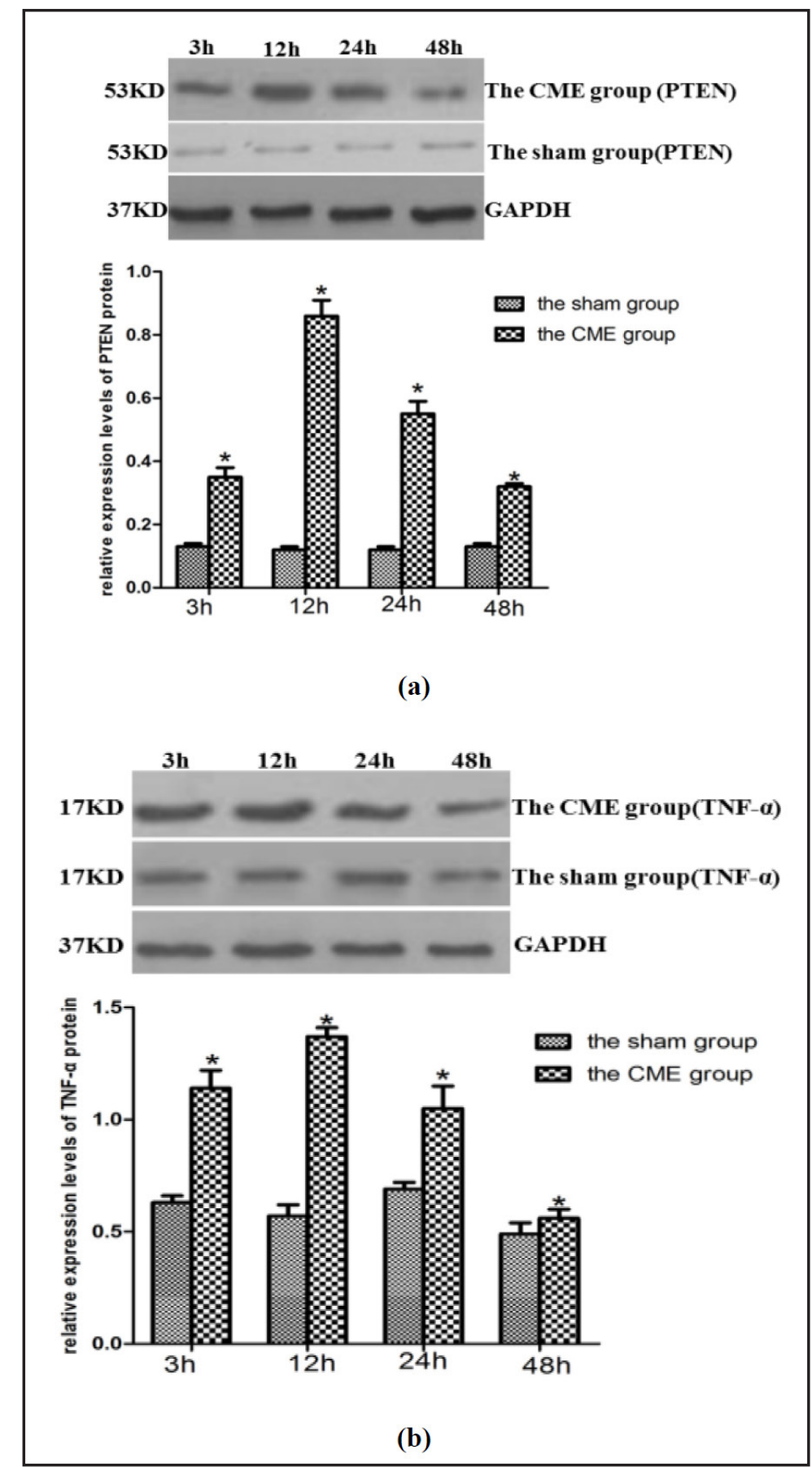

Serial changes in PTEN and TNF- $\alpha$ mRNA expression after CME

PTEN mRNA expression was significantly increased in the CME group compared with the Sham group at the corresponding stages (Fig. 3a), with similar trends for the TNF- $\alpha$ mRNA (Fig. 3b). PTEN and TNF- $\alpha$ mRNA in the CME group rapidly increased as early as $3 \mathrm{~h}$ following the surgery, increased to the highest levels at $12 \mathrm{~h}$, and then decreased continuously at $24 \mathrm{~h}$ and $48 \mathrm{~h}$ compared with the Sham group.

Serial changes in PTEN and TNF- $\alpha$ protein expression after CME

PTEN protein expression was significantly increased in the CME group compared with that in the Sham group at the corresponding stages (Fig. 4a), with a similar trend for TNF- $\alpha$ 
Fig. 5. PTEN protein expression was positively correlated with TNF- $\alpha$ protein expression $(r=0.863, p<0.001)$ and serum cTnI levels $(r=0.971, p<0.001)$ based on Pearson correlation analysis but was negatively correlated with the LVEF ( $r=-0.921, p<0.001)$.

protein expression (Fig. 4b). PTEN and TNF- $\alpha$ protein levels rapidly increased as early as $3 \mathrm{~h}$ following the surgery, increased to their highest levels at $12 \mathrm{~h}$, and then decreased continuously at $24 \mathrm{~h}$ and $48 \mathrm{~h}$ compared with the Sham group.

Pearson correlation analysis

Using Pearson correlation analysis, PTEN protein expression positively correlated with the TNF- $\alpha$ protein $(r=0.863, \mathrm{p}<0.001)$ and serum cTnI $(r=0.971, \mathrm{p}<0.001)$ levels. Additionally, PTEN protein expression negatively correlated with the LVEF $(r=-0.921$, $p<0.001$ ) (Fig. 5).

Serial changes in a variety of indicators after PTEN SIRNA treatment

Table 3 shows that PTEN siRNA treatment reduced the size of the microinfarct area (Fig. 2c), improved cardiac function (LVEF) (Fig. 6b), reduced the release of myocardial injury markers (cTnI) (Fig. 6a), and suppressed CME-induced increases in PTEN and TNF- $\alpha$ mRNA and protein expression (Fig. 6c, d, 7).

\section{Discussion}

CME is widely observed with acute coronary syndrome and is considered an iatrogenic complication following coronary interventions in clinical settings [18]. It is thought that $\mathrm{CME}$ caused by the embolization of thrombotic material and debris or the rupture of an atherosclerotic plaque generates a transient decrease in coronary blood flow followed by reactive hyperemia and myocardial systolic dysfunction. Rioufol et al. [19] demonstrated

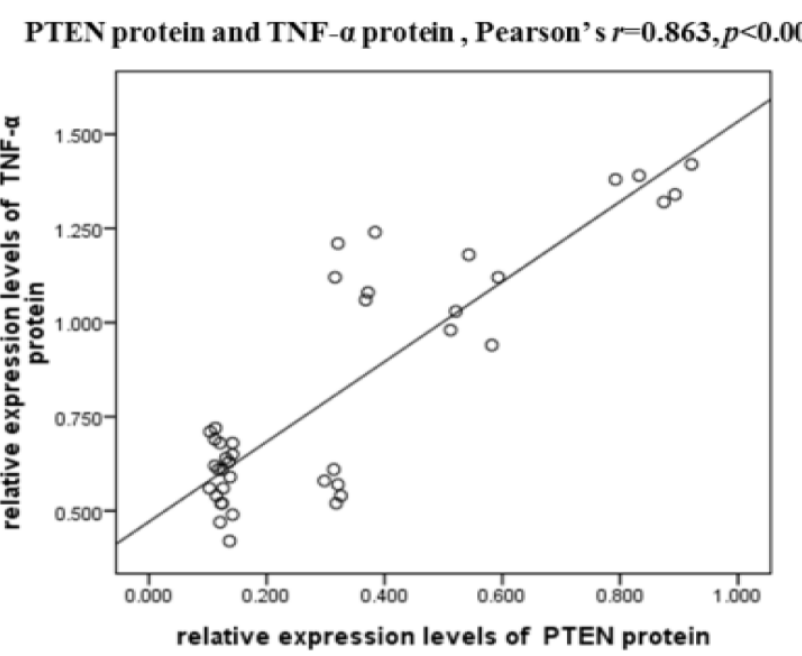

PTEN protein and cTnI, Pearson's $r=0.971, p<0.001$

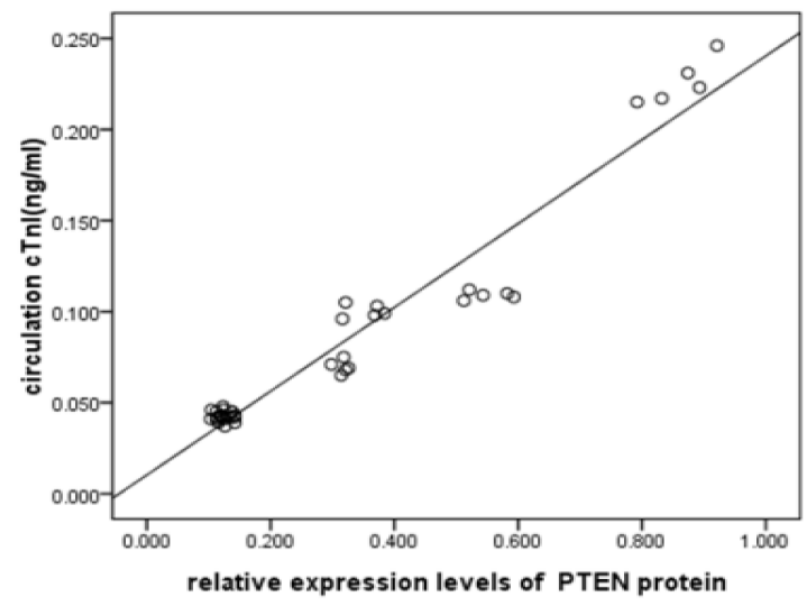

PTEN protein and LVEF, Pearson's $r=-0.921, p<0.001$

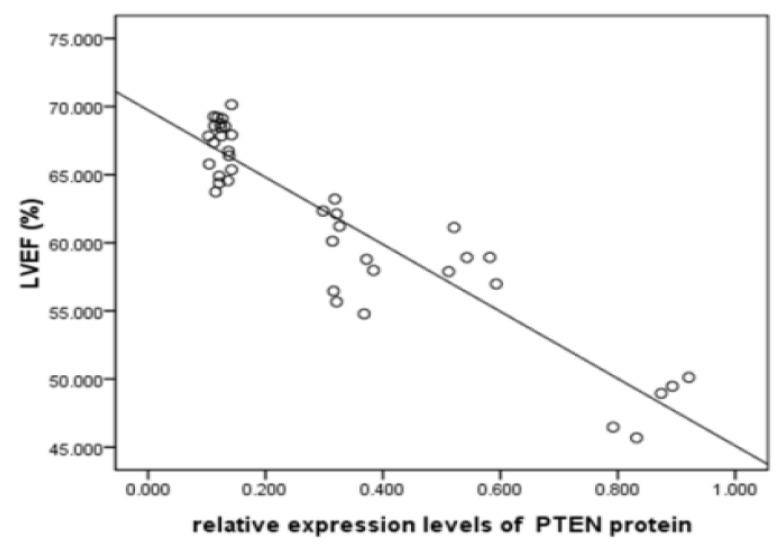


Table 3. Serial changes in a variety of indicators after PTEN siRNA treatment. Each point represents the mean (SD). a, p $<0.05$ compared with the Sham group; b, $p<0.05$ compared with CME/pre-treated with control siRNA

\begin{tabular}{lllll}
\hline Parameters & Sham & CME & PTEN siRNA & Control siRNA \\
\hline Microinfarct volume (\%) & 0.00 & $4.84(1.27)^{\mathrm{a}}$ & $2.21(1.32)^{\mathrm{ab}}$ & $4.62(1.45)^{\mathrm{a}}$ \\
LVEF (\%) & $66.76(2.45)$ & $48.14(1.95)^{\mathrm{a}}$ & $61.23(2.03)^{\mathrm{ab}}$ & $49.12(1.92)^{\mathrm{a}}$ \\
CTnI (ng/mL) & $0.042(0.003)$ & $0.226(0.013)^{\mathrm{a}}$ & $0.087(0.005)^{\mathrm{ab}}$ & $0.218(0.015)^{\mathrm{a}}$ \\
PTEN mRNA & $0.42(0.05)$ & $3.08(0.11)^{\mathrm{a}}$ & $1.65(0.08)^{\mathrm{ab}}$ & $2.98(0.12)^{\mathrm{a}}$ \\
TNF- $\alpha$ mRNA & $1.84(0.08)$ & $4.84(0.16)^{\mathrm{a}}$ & $2.36(0.12)^{\mathrm{ab}}$ & $4.63(0.17)^{\mathrm{a}}$ \\
PTEN protein & $0.23(0.03)$ & $0.77(0.07)^{\mathrm{a}}$ & $0.33(0.06)^{\mathrm{ab}}$ & $0.73(0.07)^{\mathrm{a}}$ \\
TNF- $\alpha$ protein $^{\text {TN }}$ & $0.20(0.05)$ & $0.92(0.06)^{\mathrm{a}}$ & $0.38(0.04)^{\mathrm{ab}}$ & $0.87(0.04)^{\mathrm{a}}$ \\
\hline
\end{tabular}
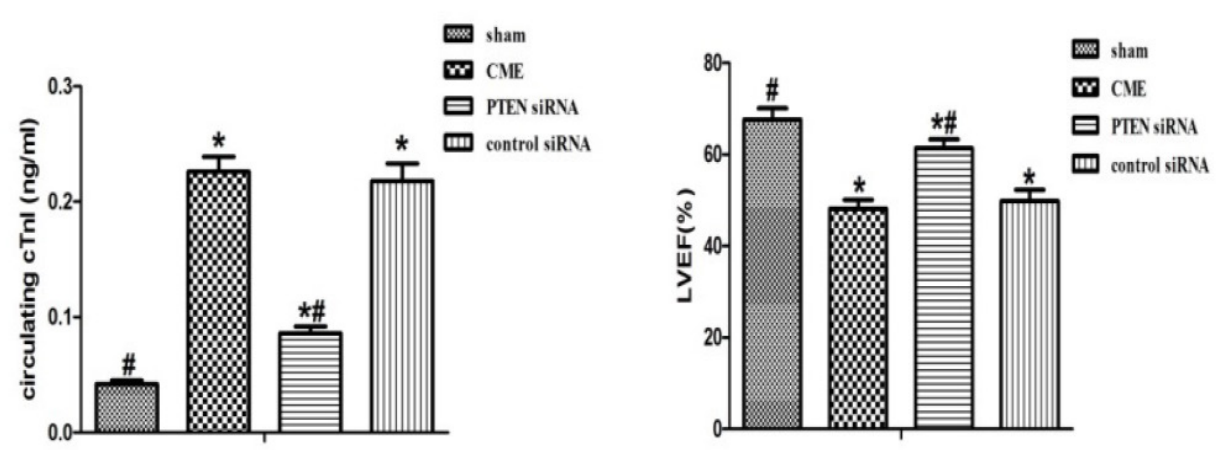

(a)

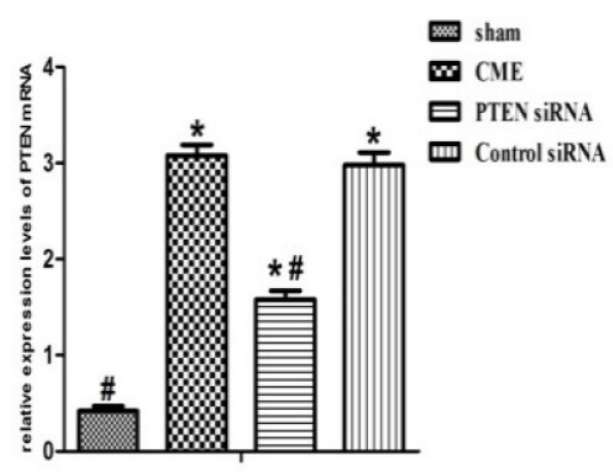

(c) (b)

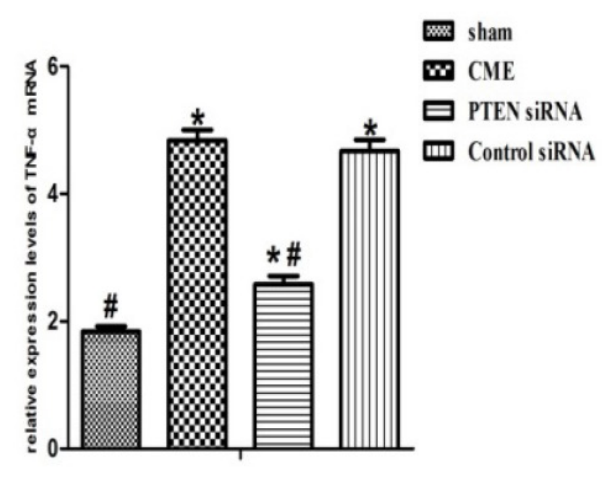

(d)

Fig. 6. Serum cTnI (a), PTEN mRNA (c), and TNF- $\alpha$ mRNA levels (d) were significantly increased in the CME and pre-treated with control siRNA groups compared with the Sham group $(p<0.05)$. Conversely, compared with the Sham group, the LVEF (\%) was significantly decreased in the CME and pre-treated with control siRNA groups (b) $(p<0.05)$. Administration of PTEN siRNA reduced PTEN mRNA, TNF- $\alpha$ mRNA, and serum cTnI levels, but not the LVEF. ${ }^{*} p<0.05$ vs. Sham; ${ }^{*} p<0.05$ vs. CME/pre-treated with control siRNA.

that the formation of atherosclerosis frequently presented with the rupture and repair of plaques. Therefore, our data further confirmed the pathophysiological manifestations of CME.

Previous studies have demonstrated that the aggregate amount of infarction involved a small area $(<5 \%)$ of microembolized myocardium in pigs or dogs, as indicated by the typical inflammatory responses with increases in TNF- $\alpha$ expression and leukocyte infiltration $[6,7$, $20,21]$. Our preliminary studies showed that a post-CME inflammatory response occurred as a result of the upregulation of TNF- $\alpha$ expression in the myocardium $[10,11]$. It has been well established that excessive TNF- $\alpha$ expression is derived from the surviving cardiomyocytes 


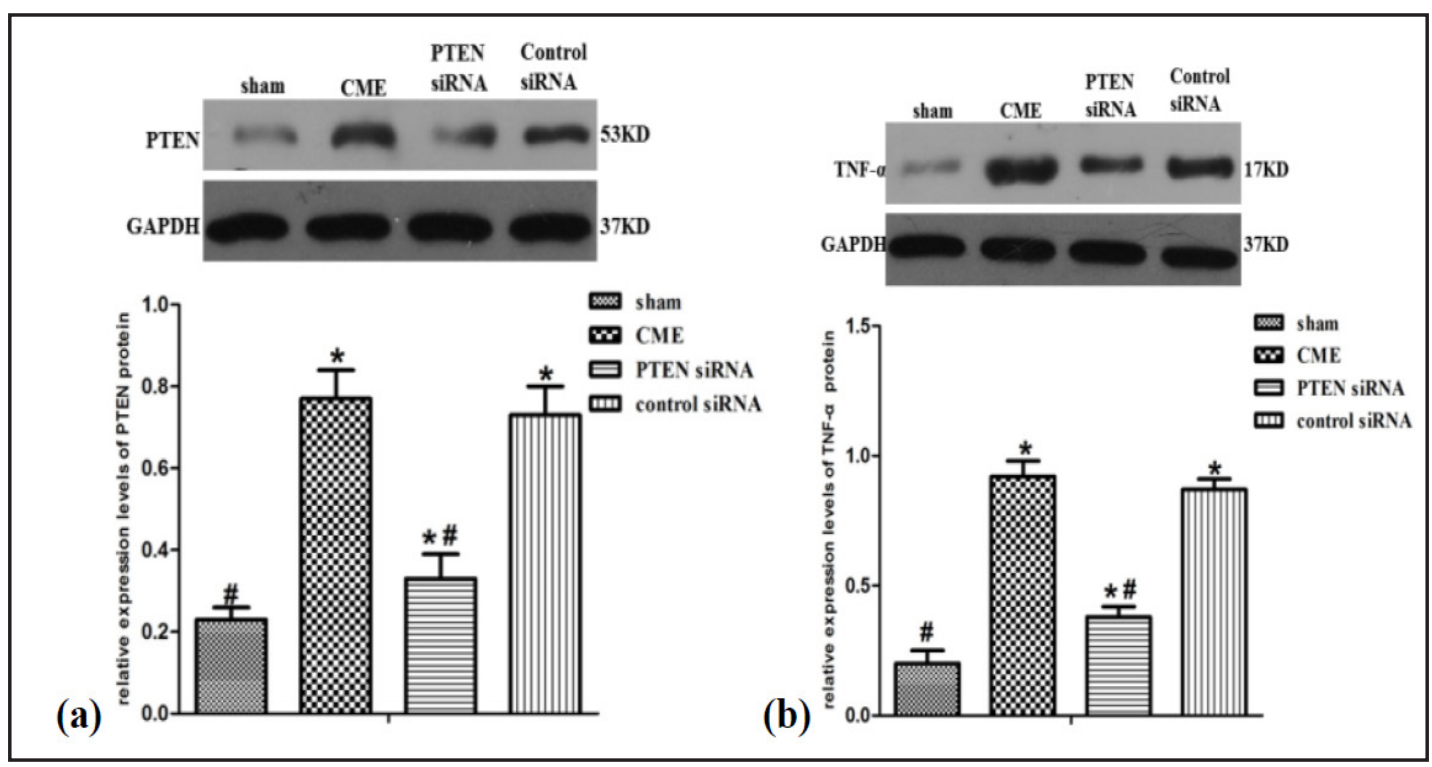

Fig. 7. PTEN and TNF- $\alpha$ protein expression levels were determined using western blot analysis (normalized to GAPDH). PTEN (a) and TNF- $\alpha$ (b) protein levels were significantly increased in the CME and pre-treated with control siRNA groups compared with the Sham group $(p<0.05)$. However, administration of PTEN siRNA reduced the PTEN and TNF- $\alpha$ protein levels. ${ }^{*} p<0.05$ vs. Sham; ${ }^{*} p<0.05$ vs. CME/pre-treated with control siRNA.

surrounding microinfarcts in autocrine or paracrine patterns [6] and is responsible for the detrimental effect on progressive contractile dysfunction [6-8, 20,22].

Our preliminary experiment revealed that TNF- $\alpha$ was activated after CME and that levels peaked $12 \mathrm{~h}$ after CME; thus, we adopted that time point in the present study. Moreover, we found that TNF- $\alpha$ expression in microembolized myocardium increased as early as 3 $\mathrm{h}$ post-CME, peaked at $12 \mathrm{~h}$, and then decreased continuously at $24 \mathrm{~h}$ and $48 \mathrm{~h}$ compared with the Sham group. This dynamic change of TNF- $\alpha$ expression was prolonged up to $48 \mathrm{~h}$ in concert with the impairment of post-CME cardiac function, suggesting that TNF- $\alpha$ was markedly elevated at the acute phase and persistently decreased during the subacute and chronic phases, which accounts for the alteration in cardiac function. Twelve hours postoperatively, malignant arrhythmia and high mortality were frequently observed. These data provide a clue regarding the optimal time point for treating extremely severe CME-related complications.

PTEN has been identified as a tumor-suppressor gene and has been associated with maintaining the balance between cell survival and cell death [23]. Growing evidence has revealed that PTEN increases myocardial contractility and is involved in post-MI remodeling by inhibiting the activation of the PI3K/AKT signaling pathway, which is ubiquitously expressed in vascular endothelial cells, vascular smooth muscle cells, cardiomyocytes, and fibroblasts [24]. Partial inactivation of PTEN reduced leukocyte infiltration into the infarcted region. Conversely, PTEN overexpression yielded the opposite effect in mice [15]. However, the inflammatory response was driven by PTEN in rats with bacterial pneumonia rather than bronchial asthma [25]. Moreover, the enhancement of PTEN expression in the myocardium promoted the inflammatory response by upregulating TNF- $\alpha$ expression in post-MI remodeling [9]. Therefore, we concluded that PTEN regulates the post-CME inflammatory response through the upregulation of TNF- $\alpha$ expression.

In our current study, PTEN enhancement of TNF- $\alpha$ activity is required for CME-induced myocardial injury, which resembles the results reported by Skyschally et al. [9], and the positive correlation was confirmed by Pearson correlation analysis. Although the involvement of PTEN in the post-CME inflammatory response was crucial for progressive contractile 
dysfunction, PTEN siRNA prevented the expression of myocardial injury markers (cTnI), reduced the microinfarct volume, restored cardiac function, and reduced PTEN and TNF- $\alpha$ mRNA and protein levels. The pernicious effect of PTEN on post-CME inflammatory injury and cardiac function deterioration was attributed to its upregulation of TNF- $\alpha$ expression.

In conclusion, PTEN plays an important role in the CME-induced inflammatory response, which can aggravate cardiac systolic dysfunction by increasing TNF- $\alpha$ expression; therefore, PTEN inhibition may have a protective effect. PTEN siRNA transfection reduced the microinfarct volume, improved cardiac function, and reduced cTnI release. Moreover, PTEN and TNF- $\alpha$ were markedly elevated at the acute phase within $12 \mathrm{~h}$ and persistently decreased during the subacute and chronic phases, which was in agreement with the impairment of post-CME cardiac function. Although our study only shows the dynamic changes of these post-CME parameters, it still gives us a potential mechanism through which to prevent CME. Further studies on the mechanism of PTEN in post-CME inflammatory injury still need to be performed.

\section{Acknowledgements}

This study was supported by the National Natural Science Foundation of China (81160046, 81260042). The authors thank Dr. Han Chen (Department of Pediatric, Wuhan Asia Heart Hospital, Wuhan, China 430000), who revised the English version of this manuscript.

\section{Disclosure Statement}

The authors declare no conflicts of interest.

\section{References}

1 Falk E: Plaque rupture with severe pre-existing stenosis precipitating coronary thrombosis. Characteristics of coronary atherosclerotic plaques underlying fatal occlusive thrombi. Br Heart J 1983;50:127-134.

2 Davies MJ, Thomas AC: Plaque fissuring---the cause of acute myocardial infarction, sudden ischaemic death, and crescendo angina. Br Heart J 1985;53:363-373.

-3 Falk E: Unstable angina with fatal outcome: Dynamic coronary thrombosis leading to infarction and/or sudden death. Autopsy evidence of recurrent mural thrombosis with peripheral embolization culminating in total vascular occlusion. Circulation 1985;71:699-708.

-4 Skyschally A, Schulz R, Erbel R, Heusch G: Reduced coronary and inotropic reserves with coronary microembolization. Am J Physiol Heart Circ Physiol 2002;282:H611-H614.

5 Dorge H, Neumann T, Behrends M, Skyschally A, Schulz R, Kasper C, Erbel R, Heusch G: Perfusioncontraction mismatch with coronary microvascular obstruction: Role of inflammation. Am J Physiol Heart Circ Physiol 2000;279:H2587-H2592.

6 Dorge H, Schulz R, Belosjorow S, Post H, van de Sand A, Konietzka I, Frede S, Hartung T, Vinten-Johansen J, Youker KA, Entman ML, Erbel R, Heusch G: Coronary microembolization: The role of tnf-alpha in contractile dysfunction. J Mol Cell Cardiol 2002;34:51-62.

-7 Thielmann M, Dorge H, Martin C, Belosjorow S, Schwanke U, van De Sand A, Konietzka I, Buchert A, Kruger A, Schulz R, Heusch G: Myocardial dysfunction with coronary microembolization: Signal transduction through a sequence of nitric oxide, tumor necrosis factor-alpha, and sphingosine. Circ Res 2002;90:807813.

8 Canton M, Skyschally A, Menabo R, Boengler K, Gres P, Schulz R, Haude M, Erbel R, Di Lisa F, Heusch G: Oxidative modification of tropomyosin and myocardial dysfunction following coronary microembolization. Eur Heart J 2006;27:875-881. 


\section{Cellular Physiology $\quad$ Cell Physiol Biochem 2014;33:1963-1974 and Biochemistry \\ Wang et al.: PTEN is Involved in CME-Induced Myocardial Inflammatory Injury}

-9 Skyschally A, Gres P, Hoffmann S, Haude M, Erbel R, Schulz R, Heusch G: Bidirectional role of tumor necrosis factor-alpha in coronary microembolization: Progressive contractile dysfunction versus delayed protection against infarction. Circ Res 2007;100:140-146.

10 Li L, Zhao X, Lu Y, Huang W, Wen W: Altered expression of pro- and anti-inflammatory cytokines is associated with reduced cardiac function in rats following coronary microembolization. Mol Cell Biochem 2010;342:183-190.

11 Li L, Li DH, Qu N, Wen WM, Huang WQ: The role of erk1/2 signaling pathway in coronary microembolization-induced rat myocardial inflammation and injury. Cardiology 2010;117:207-215.

$\checkmark 12$ Siddall HK, Warrell CE, Yellon DM, Mocanu MM: Ischemia-reperfusion injury and cardioprotection: Investigating PTEN, the phosphatase that negatively regulates PI3K, using a congenital model of PTEN haploinsufficiency. Basic Res Cardiol 2008;103:560-568.

13 Keyes KT, Xu J, Long B, Zhang C, Hu Z, Ye Y: Pharmacological inhibition of PTEN limits myocardial infarct size and improves left ventricular function postinfarction. Am J Physiol Heart Circ Physiol 2010;298:H1198-H1208.

-14 Oudit GY, Kassiri Z, Zhou J, Liu QC, Liu PP, Backx PH, Dawood F, Crackower MA, Scholey JW, Penninger JM: Loss of PTEN attenuates the development of pathological hypertrophy and heart failure in response to biomechanical stress. Cardiovasc Res 2008;78:505-514.

15 Furgeson SB, Simpson PA, Park I, Vanputten V, Horita H, Kontos CD, Nemenoff RA, Weiser-Evans MC: Inactivation of the tumour suppressor, PTEN, in smooth muscle promotes a pro-inflammatory phenotype and enhances neointima formation. Cardiovasc Res 2010;86:274-282.

16 Carlsson M, Wilson M, Martin AJ, Saeed M: Myocardial microinfarction after coronary microembolization in swine: MR imaging characterization. Radiology 2009;250:703-713.

17 Chen CH, Jiang Z, Yan JH, Yang L, Wang K, Chen YY, Han JY, Zhang JH, Zhou CM: The involvement of programmed cell death 5 (PDCD5) in the regulation of apoptosis in cerebral ischemia/reperfusion injury. CNS Neurosci Ther 2013;19:566-576.

18 Heusch G, Kleinbongard P, Bose D, Levkau B, Haude M, Schulz R, Erbel R: Coronary microembolization: From bedside to bench and back to bedside. Circulation 2009;120:1822-1836.

19 Rioufol G, Gilard M, Finet G, Ginon I, Boschat J, Andre-Fouet X: Evolution of spontaneous atherosclerotic plaque rupture with medical therapy: Long-term follow-up with intravascular ultrasound. Circulation 2004;110:2875-2880.

20 Skyschally A, Schulz R, Gres P, Konietzka I, Martin C, Haude M, Erbel R, Heusch G: Coronary microembolization does not induce acute preconditioning against infarction in pigs---the role of adenosine. Cardiovasc Res 2004;63:313-322.

-21 Arras M, Strasser R, Mohri M, Doll R, Eckert P, Schaper W, Schaper J: Tumor necrosis factor-alpha is expressed by monocytes/macrophages following cardiac microembolization and is antagonized by cyclosporine. Basic Res Cardiol 1998;93:97-107.

22 Skyschally A, Haude M, Dorge H, Thielmann M, Duschin A, van de Sand A, Konietzka I, Buchert A, Aker S, Massoudy P, Schulz R, Erbel R, Heusch G: Glucocorticoid treatment prevents progressive myocardial dysfunction resulting from experimental coronary microembolization. Circulation 2004;109:2337-2342.

23 Yamada KM, Araki M: Tumor suppressor PTEN: Modulator of cell signaling, growth, migration and apoptosis. J Cell Sci 2001;114:2375-2382.

24 Oudit GY, Sun H, Kerfant BG, Crackower MA, Penninger JM, Backx PH: The role of phosphoinositide-3 kinase and PTEN in cardiovascular physiology and disease. J Mol Cell Cardiol 2004;37:449-471.

-25 Schabbauer G, Matt U, Gunzl P, Warszawska J, Furtner T, Hainzl E, Elbau I, Mesteri I, Doninger B, Binder BR, Knapp S: Myeloid PTEN promotes inflammation but impairs bactericidal activities during murine pneumococcal pneumonia. J Immunol 2010;185:468-476. 